\title{
ON GENERAL EULERIAN INTEGRAL FORMULAS AND FRACTIONAL INTEGRATION
}

\author{
K. C. GUPTA, S. P. GOYAL AND R. K. LADDHA
}

\begin{abstract}
In the present work, we evaluate a unified Eulerian type integral whose integrand involves the product of a polynomial system and the multivariable H-function having general arguments. Our integral formula encompasses a very large number of integrals and provides interesting unifieation and extensions of several known (e.g., [1], [3], [4], [5], [9], [11], etc.) and new results. Since the integral has been given in a compact form free from infinite series, it is likely to prove useful in applications. Three special cases of the main integral (which are also sufficiently general in nature and are of interest in themselves) have also been given. Finally, the main integral formula has been expressed as a fractional integral operator to make it more useful in applications.
\end{abstract}

\section{Introduction}

Recently, a number of research workers such as Raina and Srivastava [3], Saxena and Nishimoto [5], Srivastava and Hussain [9], Srivastava and Singh [11], Srivastava and Garg [7], Gupta and Soni [1], Saigo and Raina [4], and others, have established a number of Eulerian integrals involving $\mathrm{H}$-functions of one and more variables. Also, by setting $b=x$, each of the Eulerian integrals (considered in the aforementioned works) can easily be rewritten as a fractional integral formula involving the familiar (fractional differ-integral operator ${ }_{a} D_{x}^{v}$ (defined by (4.1)).

In an attempt to unify the aforementioned results and several other results, we evaluate general Eulerian integral formula involving a product of a general class of polynomials and multivariable H-function with general arguments. This integral, besides being of very general character has been put in a compact form avoiding the occurrance of infinite series and thus making it useful in applications. Also this formula is then rewritten as a fractional integral formula involving the operator ${ }_{a} D_{x}^{-\beta}$.

The multivariable H-function occurring in this paper has been defined by Srivastava and and Panda [10, p271, Eq. (4.1)]. We shall use the following contracted notation (see

Received September 14, 1998; revised Octorber 16, 1998.

Key words and phrases. Eulerian integral formula, multivariable $\mathrm{H}$-function, general class of polynomials, orthogonal polynomials, Mellin- Barnes contour integral, Fractional integral operator. 
also $[8$, p. 251, Eq. (C.1)]:

$$
\begin{aligned}
& H\left[z_{1}, \ldots, z_{r}\right]=H \begin{array}{l}
0, N: M_{1}, N_{1} ; \ldots ; M_{r}, N_{r} \\
P, Q: P_{1}, Q_{1} ; \ldots ; P_{r}, Q_{r}
\end{array}\left[\begin{array}{c}
z_{1} \\
\vdots \\
z_{r}
\end{array} \mid\right. \\
& \left(a_{j} ; A_{j}^{\prime}, \ldots, A_{j}^{(r)}\right)_{1, P}:\left(c_{j}^{\prime}, E_{j}^{\prime}\right)_{1, P_{1}} ; \ldots ;\left(c_{j}^{(r)}, E_{j}^{(r)}\right)_{1, P_{r}} \\
& \left(b_{j} ; B_{j}^{\prime}, \ldots, B_{j}^{(r)}\right)_{1, Q}:\left(d_{j}^{\prime}, D_{j}^{\prime}\right)_{1, Q_{1}} ; \ldots ;\left(d_{j}^{(r)}, D_{j}^{(r)}\right)_{1, Q_{r}}
\end{aligned}
$$

to denote the H-function of $r$ complex variables $z_{1}, \ldots, z_{r}$. Here all the Greek latters are assumed to be positive real numbers for standardisation purposes; the definition of the multivariable $\mathrm{H}$-function will however, meaningful even if some of these quantities are zero.

The defining integral of this function, its various special cases and other details can be found in the aforementioned references. It is known that multiple Mellin-Barnes contour integral corresponding to multivariable $\mathrm{H}$-function (1.1) converges absolutely under the following conditions

$$
\begin{array}{r}
\Omega_{i}=-\sum_{j=N+1}^{P} A_{j}^{(i)}+\sum_{j=1}^{N_{i}} E_{j}^{(i)}-\sum_{j=N_{i}+1}^{P_{i}} E_{j}^{(i)}-\sum_{j=1}^{Q} B_{j}^{(i)}+\sum_{j=1}^{M_{i}} D_{j}^{(i)}-\sum_{j=M_{i}+1}^{Q_{i}} D_{j}^{(i)}>0 \\
(i=1, \ldots, r)
\end{array}
$$

$\left|\arg \left(z_{i}\right)\right|<\frac{1}{2} \Omega_{i} \pi \quad(i=1, \ldots, r)$

It is assumed throughout the present work that this function always satisfies the appropriate existence and convergence conditions given above. mials:

Srivastava [6, p.1, Eq. (1)] has also introduced the following general class of polyno-

$$
S_{n}^{m}[x]=\sum_{k=0}^{[n / m]} \frac{(-n)_{m k}}{k !} A_{n, k} x^{k}, \quad n=0,1,2, \ldots
$$

where $m$ is arbitrary positive integer and the coefficients $A_{n, k}(n, k \geq 0)$ are arbitrary constants, real or complex. On specializing these coefficients, the polynomials system (1.4) can be reduced to several classes of classical orthogonal polynomials and generalized hypergeometric polynomials (for details, see Srivastava and Singh [11]).

\section{Eulerian Integral Formula}

$$
\begin{aligned}
& \int_{a}^{b}(t-a)^{\alpha-1}(b-t)^{\beta-1} \prod_{i=1}^{T}\left\{\left(U_{i} t+V_{i}\right)^{\nu_{i}}\right\} \\
& \cdot \prod_{u=1}^{p}\left\{S_{n_{u}}^{m_{u}}\left[e_{u}(t-a)^{\sigma_{1}^{(u)}}(b-t)^{\sigma_{2}^{(u)}} \prod_{i=1}^{T}\left\{\left(U_{i} t+V_{i}\right)^{\mu_{i}^{(u)}}\right\}\right]\right\}
\end{aligned}
$$




$$
\prod_{i=1}^{S}\left\{\Gamma\left(-\theta_{i}\right) \Gamma\left(-K_{i}+\theta_{i}\right)\left[\frac{(t-a) U_{i}}{a U_{i}+V_{i}}\right]^{\theta_{i}}\right\} d \theta_{1} \ldots, d \theta_{S}
$$

amd

$$
\begin{array}{r}
\prod_{i=S+1}^{T}\left\{\left(U_{i} t+V_{i}\right)^{K_{i}}=\prod_{i=S+1}^{T}\left\{\frac{\left(b U_{i}+V_{i}\right)^{K_{i}}}{\Gamma\left(-K_{i}\right)}\right\} \cdot\left(\frac{1}{2 \pi \omega}\right)^{T-S} \int_{L_{S+1}} \cdots \int_{L_{T}}\right. \\
\prod_{i=S+1}^{T}\left\{\Gamma\left(-\theta_{i}\right) \Gamma\left(-K_{i}+\theta_{i}\right)\left[-\frac{(b-t) U_{i}}{b U_{i}+V_{i}}\right]^{\theta_{i}}\right\} d \theta_{S+1}, \ldots, d \theta_{T}
\end{array}
$$

Next, in the resulting expression so obtained, we interchange the order of integration and summation and the order of $r+T$ contour integrals and the $t$-integral which is permissible under the conditions stated. Now, evaluating the innermost $t$-integral with the help of the slightly variant form of the well-known Beta integral, and re-interpreting the multiple Mellin-Barnes contour integral in terms of the H-function of $r+T$ variables, we easily arrive at the desired result (2.1).

\section{Special cases}

Our main integral (2.1) is unified in nature and possesses manifold generalty. It acts as a key formula. Thus the multivariable H-function occurring in this integral can be suitably specialized to a remarkably wide variety of special functions (or product of several such functions) which are expressible in terms of $\mathrm{E}, \mathrm{G}$ and $\mathrm{H}$-function of one or more variables. For example, if $N=P=Q=0$, the multivariable H-function occurring in the left hand side of the integral (2.1) would reduce immediately to the product of $r$ different Fox's H-functions. Again, by suitiably specializing the coefficients $A_{n, k}$, the general class of polynomials can be reduced to a large number of orthogonal and hypergeometric polynomials. Thus using the tables listing various special cases of the H-function [8, p. 18-19] and general class of polynomials [11, p. 158-161], one can easily obtain from (2.1), a number of other integrals involving these simpler special functions and polynomials. We record below some of the special cases of (2.1) which are new and of interest in themselves:

(i) On taking $T=2$ in (2.1), we get the following interesting and relatively simpler integral formula

$$
\begin{aligned}
& \int_{a}^{b}(t-a)^{\alpha-1}(b-t)^{\beta-1}(U t+V)^{\nu}(y t+z)^{\gamma} \\
& \cdot \prod_{u=1}^{p}\left\{S_{n_{u}}^{m_{u}}\left[e_{u}(t-a)^{\sigma_{1}^{(u)}}(b-t)^{\sigma_{2}^{(u)}}(U t+V)^{\mu^{(u)}}(y t+z)^{\varepsilon^{(u)}}\right\}\right. \\
& \cdot H\left[w_{1}(t-a)^{\delta_{1}^{\prime}}(b-t)^{\delta_{2}^{\prime}}(U t+V)^{-\rho^{\prime}}(y t+z)^{-\eta^{\prime}}, \ldots,\right. \\
& \left.w_{r}(t-a)^{\delta_{i}^{(r)}}(b-t)^{\delta_{2}^{(r)}}(U t+V)^{-\rho^{(r)}}(y t+z)^{-\eta^{(r)}}\right] d t
\end{aligned}
$$




$$
\begin{aligned}
& \cdot H\left[w_{1}(t-a)^{\delta_{1}{ }^{\prime}}(b-t)^{\delta_{2}{ }^{\prime}} \prod_{i=1}^{T}\left\{\left(U_{i} t+V_{i}\right)^{-\rho_{i}{ }^{\prime}}\right\}, \ldots\right. \\
& \left.w_{r}(t-a)^{\delta_{1}^{(r)}}(b-t)^{\delta_{2}^{(r)}} \prod_{i=1}^{T}\left\{\left(U_{i} t+V_{i}\right)^{-\rho_{i}^{(r)}}\right\}\right] d t \\
& =\sum_{k_{1}=0}^{\left[n_{1} / m_{1}\right]} \cdots \sum_{k_{p}=0}^{\left[n_{p} / m_{p}\right]} \prod_{u=1}^{p}\left\{\frac{\left(-n_{u}\right)_{m_{u} k_{u}}}{k_{u} !} A_{n_{u}, k_{u}}^{(u)} e_{u}^{k_{u}}\right\} \\
& \cdot \prod_{i=1}^{S}\left\{\left(a U_{i}+V_{i}\right)^{\nu_{i}+\Sigma \mu_{i}^{(u)} k_{u}}\right\} \prod_{i=S+1}^{T}\left\{\left(b U_{i}+V_{i}\right)^{\nu_{i}+\Sigma \mu_{i}^{(u)} k_{u}}\right\}
\end{aligned}
$$

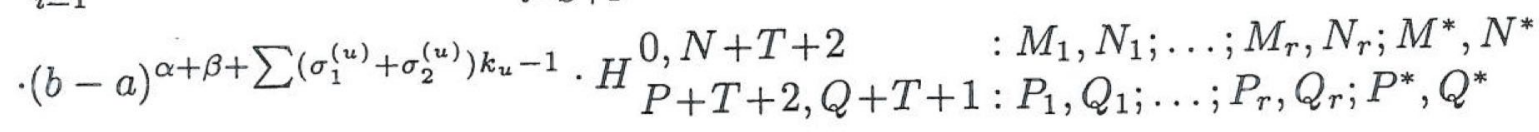

$$
\begin{aligned}
& {\left[\begin{array}{l|l}
F_{1} & \\
\vdots & F_{r} \\
I_{1} & A^{*}:\left(c_{j}^{\prime}, E_{j}^{\prime}\right)_{1, P_{1}} ; \ldots ;\left(c_{j}^{(r)}, E_{j}^{(r)}\right)_{1, P_{r}} ;-\ldots ;- \\
\vdots & \\
I_{s} & B^{*}:\left(d_{j}^{\prime}, D_{j}^{\prime}\right)_{1, Q_{1}} ; \ldots ;\left(d_{j}^{(r)}, D_{j}^{(r)}\right)_{1, Q_{r}} ;(0,1) ; \ldots ;(0,1) \\
G_{s+1} & \\
\vdots & \\
G_{T} &
\end{array}\right]}
\end{aligned}
$$

where the remaining parameters of the multivariable $H$-function occurring on left hand side of (2.1) are precisely same as those given on left hand side of (1.1)

Also in (2.1) the integers $S$ and $T$ satisfy the inequality $0 \leq S \leq T$;

$$
\begin{aligned}
& \left.\begin{array}{l}
F_{j}=w_{j}(b-a)^{\delta_{1}^{(j)}+\delta_{2}^{(j)}} \prod_{i=1}^{S}\left\{\left(a U_{i}+V_{i}\right)^{-e_{i}^{(j)}}\right\} \prod_{i=S+1}^{T}\left\{\left(b U_{i}+V_{i}\right)^{-e_{i}^{(j)}}\right\} \\
I_{k}=(b-a) U_{k} /\left(a U_{k}+V_{k}\right), G_{l}=-(b-a) U_{l} /\left(b U_{l}+V_{l}\right)
\end{array}\right\} \\
& (j=1, \ldots, r ; k=1, \ldots, S ; l=S+1, \ldots, T), \\
& A^{*}=(1+\nu_{1}+\Sigma \mu_{1}^{(\mu)} k_{u} ; \rho_{1}^{\prime}, \ldots, \rho_{1}^{(r)}, 1, \underbrace{0, \ldots, 0}_{T-1}), \ldots \\
& (1+\nu_{T}+\Sigma \mu_{T}^{(u)} k_{u} ; \rho_{T}^{\prime}, \ldots, \rho_{T}^{(r)}, \underbrace{0, \ldots, 0}_{T-1}, 1), \\
& (1-\alpha-\Sigma \sigma_{1}^{(u)} k_{u} ; \delta_{1}^{\prime}, \ldots, \delta_{1}^{(r)}, \underbrace{1, \ldots, 1}_{S}, \underbrace{0, \ldots, 0}_{T-S}) \\
& (1-\beta-\Sigma \sigma_{2}^{(u)} k_{u} ; \delta_{2}^{\prime}, \ldots, \delta_{2}^{(r)}, \underbrace{0, \ldots, 0}_{S}, \underbrace{1, \ldots, 1}_{T-S}) \\
& (a_{j} ; A_{j}^{\prime}, \ldots, A_{j}^{(r)}, \underbrace{0, \ldots, 0}_{T})_{1, P}
\end{aligned}
$$




$$
\begin{aligned}
B^{*}= & (b_{j} ; B_{j}^{\prime}, \ldots, B_{j}^{(r)}, \underbrace{0, \ldots, 0}_{T})_{1, Q}, \\
& (1+\nu_{i}+\sum \mu_{i}^{(u)} k_{u} ; \rho_{i}^{\prime}, \ldots, \rho_{i}^{(r)}, \underbrace{0, \ldots, 0}_{T})_{1, T}, \\
& (1-\alpha-\beta-\sum\left(\sigma_{1}^{(u)}+\sigma_{2}^{(u)}\right) k_{u} ; \delta_{1}^{\prime}+\delta_{2}^{\prime}, \ldots, \delta_{1}^{(r)}+\delta_{2}^{(r)}, \underbrace{1, \ldots, 1}_{T})
\end{aligned}
$$

and

$$
\begin{aligned}
& M^{*}, N^{*} \quad 1,0 ; \ldots ; 1,0 \\
& P^{*}, Q^{*} \equiv, 1 ; \ldots ; 0,1,
\end{aligned}
$$

(where the total number of parameters of the type $\begin{aligned} & 1,0 \\ & 0,1\end{aligned}$ is $T$ ).

Again the letter occurring below the bracket $\underbrace{\sim}$ at any place on the right hand side of (2.1) indicates the number of zeros/ones covered by the bracket. Thus $\underbrace{0, \ldots, 0}_{T}$ would mean $T$ zeros and so on.

Also $\Sigma \equiv \sum_{u=1}^{P}$

The conditions of validity for (2.1) are

(i) $\min _{1 \leq i \leq T}\left\{\delta_{1}^{(j)}, \delta_{2}^{(j)}, \rho_{i}^{(j)}, \sigma_{1}^{(u)}, \sigma_{2}^{(u)}, \mu_{i}^{(u)}\right\} \geq 0(j=1, \ldots, r ; u=1, \ldots, p)$ (not all zero simultaneously)

(ii) $\operatorname{Re}(\alpha)>0, \operatorname{Re}(\beta)>0, \operatorname{Re}\left(\nu_{i}\right)>0(i=1, \ldots, T)$

(iii) $\operatorname{Re}\left(\alpha+\sum_{u=1}^{p} \sigma_{1}^{(u)} k_{u}+\sum_{j=1}^{r} \delta_{1}^{(j)} \varsigma_{j}\right)>0$,

$$
\operatorname{Re}\left(\beta+\sum_{u=1}^{p} \sigma_{2}^{(u)} k_{u}+\sum_{j=1}^{r} \delta_{2}^{(j)} \varsigma_{j}\right)>0,\left(k_{u}=0,1, \ldots,\left[n_{u} / m_{u}\right]\right)
$$

where $\varsigma_{j}=\min _{1 \leq i \leq M_{j}}\left[\operatorname{Re}\left(d_{i}^{(j)} / D_{i}^{(j)}\right)\right],(j=1, \ldots, r)$

Proof. To establish the integral formula (2.1), we first expand each polynomial set occurring in its L.H.S. by using (1.4) and replace the multivariable H-function by its Mellin-Barnes contour integral $[8, \mathrm{p} .251]$. In the expression thus obtained we write

$$
\begin{aligned}
& \prod_{i=1}^{T}\left(U_{i} t+V_{i}\right)^{K_{i}}=\prod_{i=1}^{S}\left\{\left(U_{i} t+V_{i}\right)^{K_{i}}\right\} \prod_{i=S+1}^{T}\left\{\left(U_{i} t+V_{i}\right)^{K_{i}}\right\} \\
& \left(K_{i}=\nu_{i}+\sum_{u=1}^{p} \mu_{i}^{(u)} k_{u}-\sum_{j=1}^{r} \rho_{i}^{(j)} \xi_{j}\right)
\end{aligned}
$$

and express the above factors in terms of Mellin-Barnes contour integral with the help of a known results. [8, p.18, Eq. (2.6.4); p. 10, Eq. (2.6.1)], and obtain

$$
\prod_{i=1}^{S}\left\{\left(U_{i} t+V_{i}\right)^{K_{i}}\right\}=\prod_{i=1}^{S}\left\{\frac{\left(a U_{i}+V_{i}\right)^{K_{i}}}{\Gamma\left(-K_{i}\right)}\right\} \cdot\left(\frac{1}{2 \pi \omega}\right)^{S} \int_{L_{1}} \cdots \int_{L_{s}}
$$




$$
\begin{aligned}
& =\sum_{k_{1}=0}^{\left[n_{1} / m_{1}\right]} \cdots \sum_{k_{p}=0}^{\left[n_{p} / m_{p}\right]} \prod_{u=1}^{p}\left\{\frac{\left(-n_{u}\right)_{m_{u} k_{u}}}{K_{u} !} A_{n_{u}, k_{u}}^{(u)} e_{u}^{k_{u}}\right\} \\
& \cdot(a U+V)^{\nu+\Sigma \mu^{(u)} k_{u}}(b y+z)^{\gamma+\Sigma \varepsilon^{(u)} k_{u}}(b-a)^{\alpha+\beta+\sum\left(\sigma_{1}^{(u)}+\sigma_{2}^{(u)}\right) k_{u}-1} \\
& \begin{array}{r}
H^{0, N+4}: M_{1}, N_{1} ; \ldots ; M_{r}, N_{r} ; 1,0 ; 1,0 \\
P+4, Q+3: P_{1}, Q_{1} ; \ldots ; P_{r}, Q_{r} ; 0,1 ; 0,1
\end{array} \\
& {\left[\begin{array}{c|c}
F_{1}^{\prime} & A_{1}^{*}:\left(c_{j}^{\prime}, E_{j}^{\prime}\right)_{1, P_{1}} ; \ldots ;\left(c_{j}^{(r)}, E_{j}^{(r)}\right)_{1, P_{r}} ;-;- \\
\vdots & F_{r}^{\prime} \\
X & B_{1}^{*}:\left(d_{j}^{\prime}, D_{j}^{\prime}\right)_{1, Q_{1}} ; \ldots ;\left(d_{j}^{(r)}, D_{j}^{(r)}\right)_{1, Q_{r}} ;(0,1) ;(0,1) \\
Y &
\end{array}\right]}
\end{aligned}
$$

where $F_{j}^{\prime}=w_{j}(b-a)^{\delta_{1}^{(j)}+\delta_{2}^{(j)}}(a U+V)^{-\rho^{(j)}}(b y+z)^{-\eta^{(j)}}(j=1, \ldots, r), X=\frac{(b-a) U}{a U+V}$, $Y=-\frac{(b-a) y}{b y+z}$,

$$
\begin{aligned}
A_{1}^{*} \equiv & \left(1+\nu+\Sigma \mu^{(u)} k_{u} ; \rho^{\prime}, \ldots, \rho^{(r)}, 1,0\right),\left(1+\gamma+\Sigma \varepsilon^{(u)} k_{u} ; \eta^{\prime}, \ldots, \eta^{(r)}, 0,1\right), \\
& \left(1-\alpha-\Sigma \sigma_{1}^{(u)} k_{u} ; \delta_{1}^{\prime}, \ldots, \delta_{1}^{(r)}, 1,0\right),\left(1-\beta-\Sigma \sigma_{2}^{(u)} k_{u} ; \delta_{2}^{\prime}, \ldots, \delta_{2}^{(r)}, 0,1\right), \\
& \left(a_{j} ; A_{j}^{\prime}, \ldots, A_{j}^{(r)}, 0,0\right)_{1, p} \\
B_{1}^{*} \equiv & \left(b_{j} ; B_{j}^{\prime}, \ldots, B_{j}^{(r)}, 0,0\right)_{1, Q},\left(1+\nu+\Sigma \mu^{(u)} k_{u} ; \rho^{\prime}, \ldots, \rho^{(r)} ; 0,0\right), \\
& \left(1+\varepsilon+\Sigma \varepsilon^{(u)} k_{u} ; \eta^{\prime}, \ldots, \eta^{(r)}, 0,0\right), \\
& \left(1-\alpha-\beta-\Sigma\left(\sigma_{1}^{(u)}+\sigma_{2}^{(u)}\right) k_{u} ; \delta_{1}^{\prime}+\delta_{2}^{\prime}, \ldots, \delta_{1}^{(r)}+\delta_{2}^{(r)}, 1,1\right)
\end{aligned}
$$

The conditions of validity of (3.1) are easily obtainable from those given with the main integral (2.1).

Further, if we put $n_{u}=0(u=1, \ldots, p)$ for which all polynomials will reduce to unity and $\delta_{1}^{(j)}=\delta_{2}^{j)}=0(j=1, \ldots, r)$ in $(3.1)$, we arrive at the result which is in essence same as obtained recently by Srivastava and Hussain [9, p.79, Eq. (2.5)], which in turn reduces to the several other known results.

(ii) If we set $T=1, p=2, a=0, U=1$ in (2.1), we get the known result due to Gupta and Soni, [1, p.51, Eq. (2.2) for $t=1$ ], which further includes many other known results.

(iii) Further, taking $T=1, N=P=Q=0$ and $r=1$ in (2.1), we get the following interesting integral formula involving Fox's H-function:

$$
\begin{aligned}
& \int_{a}^{b}(t-a)^{\alpha-1}(b-t)^{\beta-1}(U t+V)^{\nu} \prod_{u=1}^{p}\left\{S_{n_{u}}^{m_{u}}\left[e_{u}(t-a)^{\sigma_{1}^{(u)}}(b-t)^{\sigma_{2}^{(u)}}(U t+V)^{\mu^{(u)}}\right]\right\} \\
& \left.\cdot H \begin{array}{c}
M, N\left[w(t-a)^{\delta_{1}}(b-t)^{\delta_{2}}(U t+V)^{-\rho}\right. \\
P, Q
\end{array} \begin{array}{l}
\left(C_{j}, E_{j}\right)_{1, P} \\
\left(d_{j}, D_{j}\right)_{1, Q}
\end{array}\right] d t
\end{aligned}
$$




$$
\begin{aligned}
& =\sum_{k_{1}=0}^{\left[n_{1} / m_{1}\right]} \cdots \sum_{k_{p}=0}^{\left[n_{p} / m_{p}\right]} \prod_{u=1}^{p}\left\{\frac{\left(-n_{u}\right)_{m_{u} k_{u}}}{k_{u} !} A_{n_{u}, k_{u}}^{(u)} e_{u}^{k_{u}}\right\} \\
& \cdot(a U+V)^{\nu+\Sigma \mu^{(u)} k_{u}}(b-a)^{\alpha+\beta+\Sigma\left(\sigma_{1}^{(u)}+\sigma_{2}^{(u)}\right) k_{u}-1}
\end{aligned}
$$

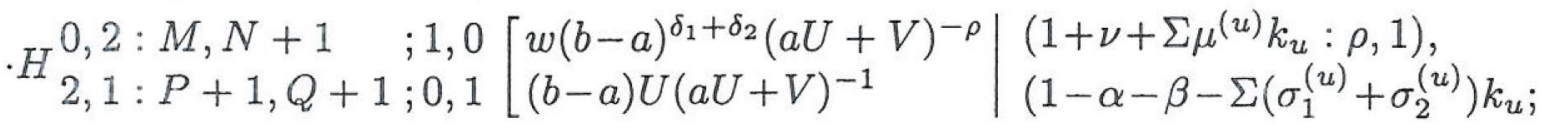

$$
\begin{aligned}
& \left(1-\alpha-\Sigma \sigma_{1}^{(u)} k_{u} ; \delta_{1}, 1\right):\left(1-\beta-\Sigma \sigma_{2}^{(u)} k_{u} ; \delta_{2}\right),\left(c_{j}, E_{j}\right)_{1, p} ;- \\
& \left.\delta_{1}+\delta_{2}, 1\right) \quad:\left(d_{j}, D_{j}\right)_{1, Q},\left(1+\nu+\Sigma \mu^{(u)} k_{u}, \rho\right) ;(0,1)
\end{aligned}
$$

where ${ }_{P, Q}^{M, N}[w]$ stands for the Fox's H-function (see, e.g., [8, p. 10]) and the function of occurring on the right-hand side is the H-function of two variables (for details, see, e.g., $[8$, p. 83].

The conditions of validity of (3.2) are easily obtainable from those given with the main integral (2.1).

Further, on putting $\delta_{1}=\delta_{2}=0$ and $n_{u}=0(u=1, \ldots, p)$ in (3.2), we arrive at a known result due to Saxena and Nishimoto [5, p. 69, Eq. (4.1)].

(iv) Next, on reducing the H-function occurring in the left-hand side of (3.2) to unity, we get the following interesting result after a little simplification

$$
\begin{aligned}
& \int_{a}^{b}(t-a)^{a-1}(b-t)^{\beta-1}(U t+V)^{\nu} \prod_{u=1}^{p}\left\{S_{n_{u}}^{m_{u}}\left[e_{u}(t-a)^{\sigma_{1}^{(u)}}(b-t)^{\sigma_{2}^{(u)}}(U t+V)^{\mu^{(u)}}\right]\right\} d t \\
= & \sum_{k_{1}=0}^{\left[n_{1} / m_{1}\right]} \cdots \sum_{k_{p}=0}^{\left[n_{p} / m_{p}\right]} \prod_{u=1}^{p}\left\{\frac{\left(-n_{u}\right)_{m_{u} k_{u}}}{k_{u} !} A_{n_{u}, k_{u}}^{(u)} e_{u}^{k_{u}}\right\} \\
& \cdot(a U+V)^{\nu+\Sigma \mu^{(u)} k_{u}}(b-a)^{\alpha+\beta+\Sigma\left(\sigma_{1}^{(u)}+\sigma_{2}^{(u)}\right) k_{u}-1} \\
& \cdot B\left(\alpha+\Sigma \sigma_{1}^{(u)} k_{u}, \beta+\Sigma \sigma_{2}^{(u)} k_{u}\right) \\
& \cdot{ }_{2} F_{1}\left[\begin{array}{l}
\alpha+\Sigma \sigma_{1}^{(u)} k_{u},-\nu-\Sigma \mu^{(u)} k_{u} \\
\alpha+\beta+\Sigma\left(\sigma_{1}^{(u)}+\sigma_{2}^{(u)}\right) k_{u}
\end{array} ;-\frac{(b-a) U}{a U+V}\right]
\end{aligned}
$$

Further, setting $\nu=-\alpha-\beta, U=\lambda-\mu, V=(1+\mu) b-(1+\lambda) a$ and $\mu^{(u)}=0$, $\sigma_{1}^{(u)}=1, \sigma_{2}^{(u)}=-1(u=1, \ldots, p)$ in (3.3), we arrive at the result recently obtained by Saigo and Raina [4, Eq. (2.1)]. Finally, taking $T=0$, and $p=1$ with $\sigma_{1}^{\prime}=0, \sigma_{2}^{\prime}=1$ in (2.1), we arrive at the integral formula evaluated earlier by Srivastava and Singh [11, p. 166 Eq. (2.2)]. Another result by Srivastava and Garg [7, p. 688, Eq. (2.1)] is also contained in (2.1) as a special case. 


\section{Application}

We can express our Eulerian integral formula as a fractional integral formula involving the operator ${ }_{a} D_{x}^{-\beta}$ (see [2]) defined by

$$
\begin{aligned}
{ }_{a} D_{x}^{v}\{f(x)\} & =\frac{1}{\Gamma(-v)} \int_{a}^{x}(x-t)^{-v-1} f(t) d t \quad(a \in R, \operatorname{Re}(v)<0) \\
& =\frac{d^{m}}{d x^{m}}{ }_{a} D_{x}^{\nu-m}\{f(x)\} \quad(0 \leq \operatorname{Re}(v)<m, m \in N)
\end{aligned}
$$

provided that the integral exists.

In fact, when $a=0$, the operator $D_{x}^{v}={ }_{0} D_{x}^{v}$ corresponds to the classical RiemannLiouville derivative (or integral) of order $v$ (or $-v$ ). Morever, when $a \rightarrow \infty$, the equation (4.1) may be indentified with the definition of the familiar Weyl fractional derivative (or integral) of order $v$ (or- $v$ )

Now, for $b=x$ and $\sigma_{1}^{(u)}=\sigma_{2}^{(u)}=0, \delta_{1}^{(j)}=\delta_{2}^{(j)}=0(u=1, \ldots, p ; j=1, \ldots, r)$, each of the Eulerian integral formula (2.1), (3.1) (3.2) and (3.3) can be written easily as a fractional integral formula involving the operator ${ }_{a} D_{x}^{-\beta}$. Thus, for example, our integral formula (2.1) yields the following result which is valid under the conditions stated already (with, of course, $b=x$ ) with (2.1):

$$
\begin{aligned}
& { }_{a} D_{x}^{-\beta}\left[(x-a)^{\alpha-1} \prod_{i=1}^{T}\left\{\left(U_{i} x+V_{i}\right)^{\nu_{i}}\right\} \prod_{u=1}^{p}\left\{S_{n_{u}}^{m_{u}}\left[e_{u} \prod_{i=1}^{T}\left\{\left(U_{i} x+V_{i}\right)^{\mu_{i}^{(u)}}\right\}\right]\right\}\right. \\
& H\left[w_{1} \prod_{i=1}^{T}\left\{\left(U_{i} x+V_{i}\right)^{-\rho_{i}^{\prime}}\right\}, \ldots, w_{r} \prod_{i=1}^{T}\left\{\left(U_{i} x+V_{i}\right)^{-\rho_{i}^{(r)}}\right\}\right] \\
& =\frac{1}{\Gamma(\beta)} \sum_{k_{1}=0}^{\left[n_{1} / m_{1}\right]} \cdots \sum_{k_{p}=0}^{\left[n_{p} / m_{p}\right]} \prod_{u=1}^{p}\left\{\frac{\left(-n_{u}\right)_{m_{u} k_{u}}}{k_{u} !} A_{n_{u}, k_{u}}^{(u)} e_{u}^{k_{u}}\right\} \\
& \cdot \prod_{i=1}^{S}\left\{\left(a U_{i}+V_{i}\right)^{\nu_{i}+\Sigma \mu_{i}^{(u)} k_{u}}\right\} \prod_{i=S+1}^{T}\left\{\left(x U_{i}+V_{i}\right)^{\nu_{i}+\Sigma \mu_{i}^{(u)} k_{u}}\right\}(x-a)^{\alpha+\beta-1} \\
& \cdot H_{P+T+2, Q+T+1: P_{1}, Q_{1} ; \cdots ; P_{r}, Q_{r} ; P^{*}, Q^{*}}^{0, N+T+2}\left[\begin{array}{l|l}
F_{1}^{\prime \prime} & \\
\vdots & \\
F_{r}^{\prime \prime} & A^{* *}: \cdots \\
I_{1} & \\
\vdots & \\
I_{s} & B^{* *}: \cdots \\
G_{s+1} & \\
\vdots & \\
G_{T} &
\end{array}\right]
\end{aligned}
$$

where the remaining parameters of the multivariable H-function are same as those men- 
tioned with (2.1). Also

$$
\begin{aligned}
& F_{j}^{\prime \prime}=W_{j} \prod_{i=1}^{S}\left\{\left(a U_{i}+V_{i}\right)^{-e_{i}^{(j)}}\right\} \prod_{i=S+1}^{T}\left\{\left(x U_{i}+V_{i}\right)^{-e_{i}^{(j)}}\right\} \text { and } \\
& I_{k}(k=1, \ldots, S), G_{l}(l=S+1, \ldots, T) \text { are given by (2.2) with } b=x .
\end{aligned}
$$

Again

$$
\begin{aligned}
& A^{* * *}=(1+\nu_{1}+\Sigma \mu_{1}^{(\mu)} k_{u} ; \rho_{1}^{\prime}, \ldots, \rho_{1}^{(r)}, 1, \underbrace{0, \ldots, 0}_{T-1}), \ldots, \\
&(1+\nu_{T}+\Sigma \mu_{T}^{(u)} k_{u} ; \rho_{T}^{\prime}, \ldots, \rho_{T}^{(r)}, \underbrace{0, \ldots, 0}_{T-1}, 1) \\
&(1-\alpha-\underbrace{0, \ldots, 0}_{r}, \underbrace{1, \ldots, 1}_{S}, \underbrace{0, \ldots, 0}_{T-S}),(1-\beta ; \underbrace{0, \ldots, 0}_{r+S}, \underbrace{1, \ldots, 1}_{T-S}), \\
&(a_{j} ; A_{j}^{\prime}, \ldots, A_{j}^{(r)}, \underbrace{0, \ldots, 0}_{T})_{1, P}, B^{* *}= \\
&(b_{j} ; B_{j}^{\prime}, B_{j}^{(r)}, \underbrace{0, \ldots, 0}_{T})_{1, Q},(1+\nu_{i}+\Sigma \mu_{i}^{(u)} k_{u} ; \rho_{i}^{\prime}, \ldots, \rho_{i}^{(r)}, \underbrace{0, \ldots, 0}_{T})_{1, T}, \\
&(1-\alpha-\beta ; \underbrace{0, \ldots, 0}_{r}, \underbrace{1, \ldots, 1}_{T})
\end{aligned}
$$

\section{Acknowledgements}

The authors are thankful to Professor H. M. Srivastava, University of Victoria, for his useful suggestions during the preparation of the paper. The first- and third-named authors are also thankful to the University Grants Commission, New Delhi, for providing necessary financial assistance for the present work.

\section{References}

[1] K. C. Gupta and R. C. Soni, On unified integrals, J. Indian Math. Soc. (N.S.) 55 (1990), 49-58.

[2] K. S. Miller and B. Ross, An introduction to the Fractional Calculus and Fractional Differential Equations, John Wiley and Sons, New York, 1993.

[3] R. K. Raina and H. M. Srivastava, Evaluation of a certain class of Eulerian integrals, J. Phys. A: Math. Gen. 26 (1993), 691-696.

[4] M. Saigo and R. K. Raina, Evaluation of certain integrals in terms of generalized Kampé de Fériet function and Lauricella function $F_{D}^{(n)}$, Tamkang J. Math. 26 (1995), 41-47.

[5] R. K. Saxena and K. Nishimoto, Fractional integral formula for the H-function, J. fractional Calculus 6 (1994), 64-75.

[6] H. M. Srivastava, A contour integral involving Fox's H-function, Indian J. Math. 14 (1972), $1-6$. 
[7] H. M. Srivastava and M. Garg, Some integrals involving a general class of polynomials and the multivariable H-function, Rev. Roumaine Phys. 32 (1987), 685-692.

[8] H. M. Srivastava, K. C. Gupta and S. P. Goyal, The H-Functions of One and Two Variables with Applications, South Asian Publ., New Delhi and Madras, 1982.

[9] H. M. Srivastava and M. A. Hussain, Fractional integration of the H-function of several variables, Comput. Math Appl. 30 (1995), 73-85.

[10] H. M. Srivastava and R. Panda, Some bilateral generating functions for a class of generalized hypergeometric polynomials, J. Reine Angew. Math. 283/284 (1976), 265-274.

[11] H. M. Srivastava and N. P. Singh, The integration of multivariable H-function with a general class of polynomials, Rend. Circ. Mat. Palermo 32, (1983), 157-187.

Emeritus Fellow (University Grants Commission), Department of Mathematics, M. R. Engineering College, Jaipur 302017, Rajasthan, India.

Department of Mathematics, University of Rajasthan, Jaipur 302004, Rajasthan, India.

Department of Mathematics, M. L. V. Government College, Bhilwara 311001, Rajasthan, India. 